\title{
Metric Features of a Dipolar Model
}

\author{
Mario Casartelli ${ }^{1,2, \dagger}$, Luca Dall’Asta ${ }^{3, \ddagger}$, Enrico Rastelli ${ }^{2,4}$ and Sofia Regina ${ }^{2}$
}

November 8, 2018

\begin{abstract}
The lattice spin model, with nearest neighbor ferromagnetic exchange and long range dipolar interaction, is studied by the method of time series for observables based on cluster configurations and associated partitions, such as Shannon entropy, Hamming and Rohlin distances. Previous results based on the two peaks shape of the specific heat, suggested the existence of two possible transitions. By the analysis of the Shannon entropy we are able to prove that the first one is a true phase transition corresponding to a particular melting process of oriented domains, where colored noise is present almost independently of true fractality. The second one is not a real transition and it may be ascribed to a smooth balancing between two geometrical effects: a progressive fragmentation of the big clusters (possibly creating fractals), and the slow onset of a small clusters chaotic phase. Comparison with the nearest neighbor Ising ferromagnetic system points out a substantial difference in the cluster geometrical properties of the two models and in their critical behavior.
\end{abstract}

${ }^{1}$ Dipartimento di Fisica dell'Università - Parco Area Scienze 7a, 43100 PR (Italy)

${ }^{2}$ CNR - Istituto Nazionale di Fisica della Materia, Parma 43100 PR (Italy)

${ }^{3}$ Laboratoire de Physique Théorique, Université de Paris-Sud, 91405 ORSAY (France)

${ }^{4}$ CNR - IMEM (Parma)

$\dagger$ Electronic address: casartelli@fis.unipr.it

$\ddagger$ Electronic address: luca.dallasta@th.u-psud.fr 


\section{Introduction}

There is a growing literature illustrating the conceptual and practical relevance of two dimensional (2D) systems with long range interactions (see [1] [2] and references therein). The model we consider here is an Ising model on a square lattice, with both nearest neighbor (NN) ferromagnetic exchange interaction, and long range dipolar interactions decaying as $r^{-3}$ among all pairs in the lattice ( $r$ being the distance). Spins are supposed to be perpendicular to the lattice plane. We shall denote PFD such a perpendicular $(\mathrm{P})$ ferromagnetic (F) dipolar (D) system. This model shows a very interesting thermodynamic behavior that results, at low temperature, in the presence of regularly shaped stripes of upwards and downward spins, and, at increasing temperature, in a complex onset of disorder until the usual random paramagnetic phase occurs. It has been supposed that one or more transitions could take place in the region between the ordered and paramagnetic phases. In particular, Ifti and coworkers [2] studied by numerical Monte Carlo (MC) simulations the behavior of specific heat, obtaining a curve with a sharp peak at temperature $T_{1}$ and a broad maximum in the region at $T_{2} \sim 2 T_{1}$. The authors suggested the existence of two possible phase transitions, the former related to the melting of the stripes, the latter to the occurrence of the paramagnetic phase. We shall study this item by MC simulation introducing a new method of investigation that seems to disprove this conjecture, in favor of a single phase transition.

Since the pioneering works by Peierls and Griffith [6], the shape and distribution of the magnetic clusters have been suggested to be a significant geometrical signature of 2D models. The problem is to give quantitative estimates and qualitative connections, beside visual inspection, between the cluster features and the thermodynamic behavior of the system. To this end, we shall extend to PFD an analysis already tested in other contexts, such as microcanonical and canonical Ising models, or self organized criticality (see [3] [4][5]). The basic tool is a map between the space $\mathcal{C} \equiv \mathcal{C}(\mathbf{M})$ of configurations on the lattice $\mathbf{M}$ and a "partition space" $\mathcal{Z} \equiv \mathcal{Z}(\mathbf{M})$, defined by the correspondence between homogeneous connected clusters and subsets of the lattice. When a dynamical simulation is performed on M, we look for possible meaningful relations between geometrical and dynamical features of quantities in $\mathcal{C}$ and $\mathcal{Z}$ and the physically relevant (thermodynamic) properties. This may be done by a time series analysis of observables related to the metric properties of $\mathcal{C}$ and $\mathcal{Z}$. The method is very general, and its efficiency consists precisely in giving indications not exclusively tailored on the model, making possible comparisons with other systems and other dynamics.

The Shannon entropy, for instance, points out the order-disorder transition by a sudden change of its slope as a function of temperature. Since the entropy continuously depends on the cluster measure distribution, this transition may be read as a topological breakdown driven by channels joining the stripped domains of the ground state. On the contrary, the order-disorder transition in the NN Ising model (studied in [4]) is driven by a fractal fragmentation of the clusters, leading to a sharp increase of entropy. Standard deviations, in both models, develop a singularity. It seems therefore that the analysis of this quantity can give information about the kind of incoming disorder. Further information can be obtained from time series of distances in $\mathcal{C}(\mathbf{M})$ and $\mathcal{Z}(\mathbf{M})$, from their standard deviation and from the analysis of power spectra, showing the dependence of "color exponents" on temperature. 
In addition, for PFD, a careful examination of clusters proves to be useful in recovering, along new and more efficient lines, previously introduced parameters and criteria [1] [2]. Notations, definitions and elementary properties of $\mathcal{C}(\mathbf{M})$ and $\mathcal{Z}(\mathbf{M})$, as well as Shannon entropy, Hamming and Rohlin distances, are recalled in the Appendix, with some mathematical details.

\section{The Model}

The Hamiltonian of the 2D perpendicular Ising ferromagnet with dipolar interactions (PFD model), is:

$$
\mathcal{H}=-J \sum_{<\mathbf{k}, \mathbf{m}>} s_{\mathbf{k}} s_{\mathbf{m}}+g \sum_{\mathbf{m} \neq \mathbf{k}} \frac{s_{\mathbf{k}} s_{\mathbf{m}}}{r_{\mathbf{k m}}^{3}}
$$

where $s_{\mathbf{k}}$ is the usual spin variable assuming values \pm 1 in the lattice $\mathbf{M}$ of size $N=L \times L$. The first sum is restricted to NN pairs, while the second sum is over all pairs. The distance $r$ is between all sites, taking into account also sites of periodically iterated copies of the lattice, up to the convergence of such sums [7]. The correspondence with the lattice $\mathbf{M}$ equipped with the binary alphabet $\mathbf{K} \equiv\{0,1\}$ and knots labelled by a couple of indexes running from 1 to $L$ is obvious. Starting from such $\mathbf{M}$ and $\mathbf{K}$, the mathematical apparatus described in the Appendix I can be developed. In particular, one may introduce the configuration space $\mathcal{C}$ provided with the Hamming distance $d_{H}$, and the cluster partition space $\mathcal{Z}$ with the Rohlin distance $d_{R}$.

The exchange constant $J$ in (1) and the temperature $T$ will be given in $g$ units. A well established result (see [1][2]) is that, for $J>0.854$ the ground state is characterized by striped domains of up and down spins, with a trivial degeneracy corresponding to their vertical or horizontal orientation. The stripe width $h$ increases as $J$. We shall assume the value $J=8.9$, corresponding to $h=8$ lattice spacings.

The specific heat $C_{V}$, as shown in Fig.1 versus temperature $T$ for a lattice of size $L=32$, coincides with the same quantity shown in Fig.3 of reference [1]. It is characterized by two peaks: a sharp one at $T=T_{1} \simeq 5$, and a broad one at $T=T_{2} \simeq 10$. When the lattice size is increased, the first peak becomes sharper and its height increases, while the second one remains unchanged, and both are slightly moved to lower temperatures [1][2]. The temperature interval of the first peak is denoted $\Delta T_{1}$. In the range $0<T<2, C_{V}$ does not move from 0 and stripes remain very stable. For $2<T<T_{1}$, where $C_{V}$ shows a sudden rise, the jagged outline of the stripe takes place gradually. For $T_{1}<T<T_{2}$ stripes are replaced by two big clusters, with unstable appearance of small fragments ("islands"). The relevance (in number and size) of such islands increases until the breakdown of big clusters occurs, approaching $T_{2}$. Finally, there is a progressive fragmentation into smaller and smaller clusters. However, up to $T=16$, completely chaotic configurations do not occur. A short summary of this process appears in Fig.2. In order to clarify the nature of these phases, we study the link between geometrical and dynamical behavior in the whole range of temperatures.

We recall that similar methods have been previously used [4] to investigate the Ising model (i.e. $g=0$ and $J=1$ in (1)). As the phase transition is approached, at temperature 


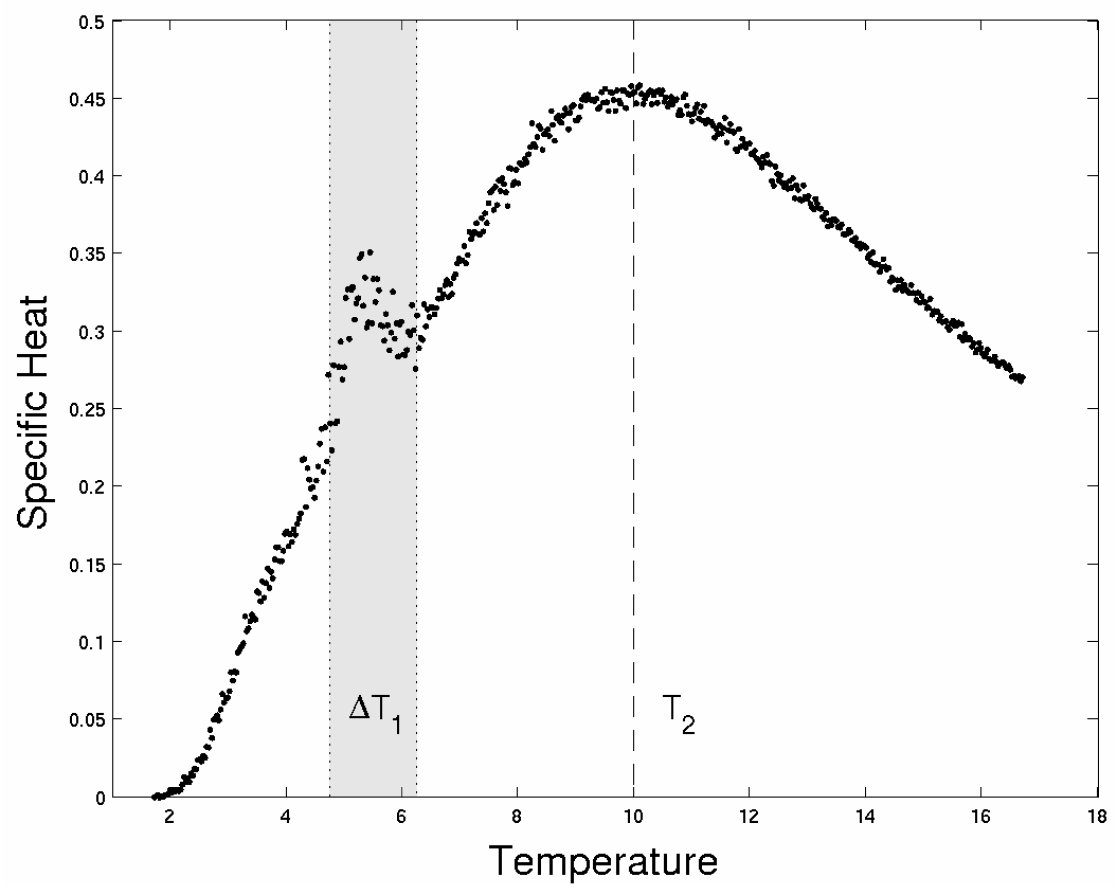

Figure 1: Specific Heat $C_{V}, L=32$, mean value over two i.c..
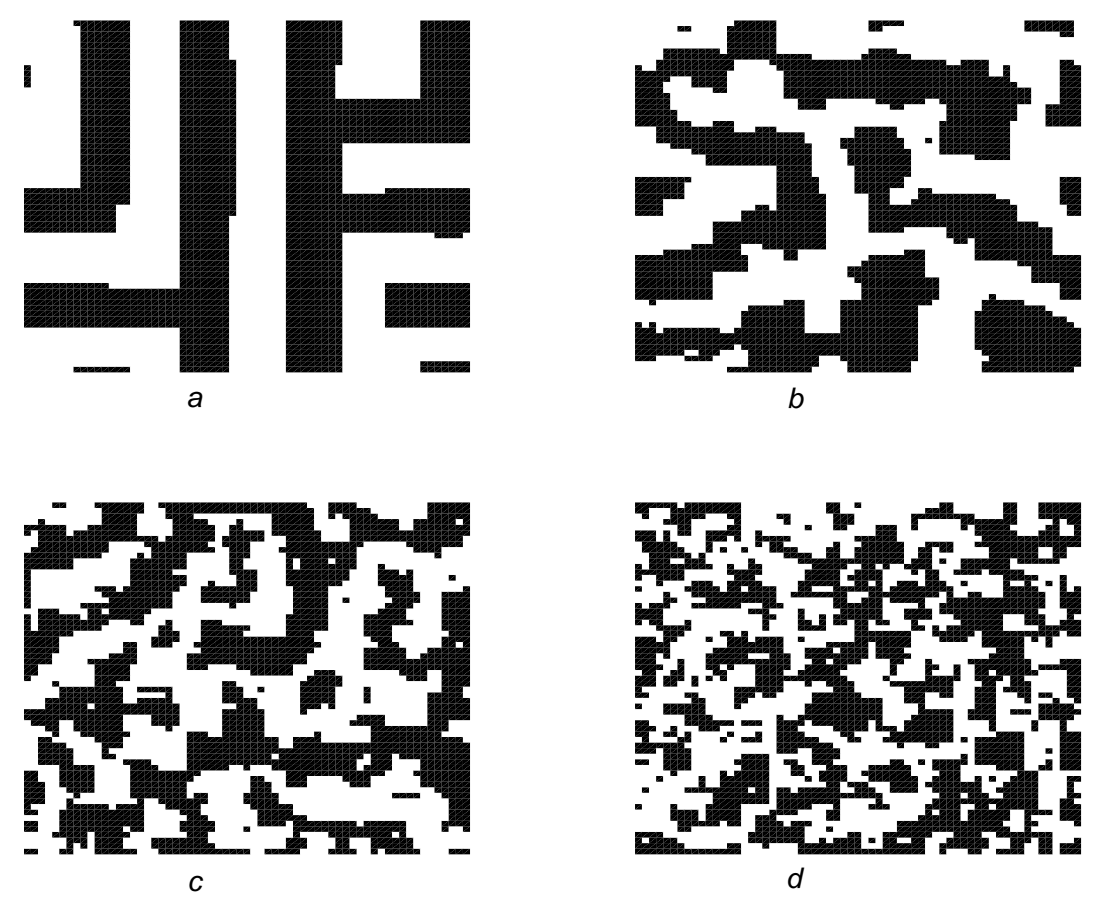

Figure 2: Configurations a, b, c, and d correspond to temperatures $\mathrm{T}=3,6,9$ and 14 respectively. 
$T_{c}$, a sudden onset of fractal structure for the magnetization cluster distribution occurs, with a singular behavior of parameters like Shannon entropy or Hamming and Rohlin distances. For instance, the standard deviation of the Shannon entropy (see Appendix) along the orbit shows a very neat peak, proving the onset of time instability for the configuration orbit at $T_{c}$ [4]. All this was independent of the evolution rule (both Metropolis and deterministic dynamics were used). One wonder whether the presence of a competitive long range interaction in the PFD model will confirm or destroy this pattern.

The question is furtherly justified by the conjecture that in a purely dipolar model, long range interactions do not influence the universality class of the Ising antiferromagnet [12]. Is it reasonable to extend this conjecture to the relation between PFD model and Ising ferromagnet? The problem is not trivial since, in such case, interactions are competitive. We shall try to answer on the basis of geometrical considerations.

\section{Numerical Experiments}

In our Monte Carlo (MC) simulations, we shall adopt the well known method based on Ewald sums [7]. This consists in considering a very large system which can be refolded into a smaller one with a renormalized coupling constant. The evolution rule is the standard Metropolis algorithm [13], where the temperature is controlled by the flip probability.

Other general data about numerical experiments are the following:

- Size: simulations have been mostly performed at $L=32$ and 64, with many consistency checks. Of course, larger values of $L$ would be expedient, in particular to control finite size effects. However, not only dipolar interactions imply a sudden rise of computing time, but there are prohibitive difficulties in handling data at increasing $L$ for entropy and Rohlin distances (see Appendix).

- Thermalization: $10^{4} \mathrm{MC}$ steps are disregarded to reach equilibrium. The simulation at temperature $T+\delta T$ uses, as starting configuration, the last thermalized configuration at temperature $T$.

- Time average interval: $\tau=2 \times 10^{4}$ steps after thermalization, that ensures a good stabilization. Therefore, for a time series $X \equiv\left\{x_{k}\right\}, \quad k=0, \ldots, \tau$, , the computed time average

$$
<x>_{\tau}=\frac{1}{\tau+1} \sum_{k=0}^{\tau} x_{k}
$$

(i.e. the usual MC thermal average) will be simply noted $\langle x\rangle$, as in the limit $\tau \rightarrow \infty$. An example of time series for Shannon Entropy is given in Fig.3, with the histogram of occurrences.

- Temperature range: simulations have been performed for $2<T<16$. This range has been sampled in two main ways: 100 values with several initial conditions (i.c.), or 500 values with two i.c. The two approaches led to consistent results. 

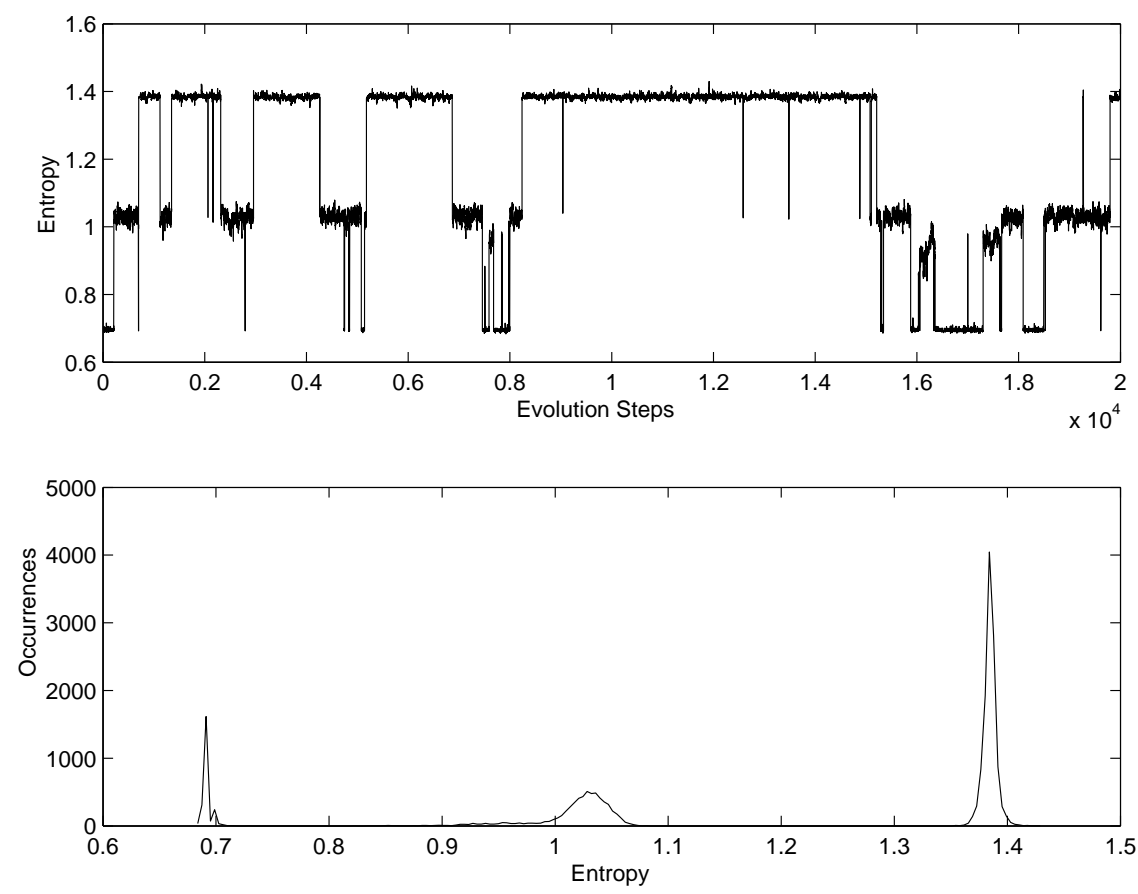

Figure 3: Entropy Time Series (above) and histogram of occurrences (below). Here $T=$ 5.15 and $L=32$.

A way to look at the meaning of time averages and their reliability consists in looking at correlations: for a time series $X \equiv\left\{x_{k}\right\}, \quad k=0, \ldots, \tau$, where $\langle x\rangle$ is the mean value, the self-correlation coefficient $\operatorname{Corr}(X, m)$ is defined as usual (see e.g. [14])

$$
\operatorname{Corr}(X, m)=\frac{\sum_{k=0}^{\tau-m}\left(x_{k+m}-<x>\right)\left(x_{k}-<x>\right)}{\sum_{k=0}^{\tau}\left(x_{k}-<x>\right)^{2}} .
$$

This coefficient displays how long an evolving quantity keeps the memory of its past, measured by the lag $m$. It indicates therefore how a time (or thermal) average is built up. For instance, in Fig.4 the self-correlation $\operatorname{Corr}(H, m)$ for the entropy time series is shown (qualitatively similar results hold for the Rohlin and Hamming distances). Fig.4 says that, apart the trivial correlation due to the freezing at low temperature, $\operatorname{Corr}(H, m)$ is sensitive to the transition at $T_{1}$, and rapidly vanishes elsewhere, particularly at increasing temperature.

Note that, below $T_{1}$, when the stripe boundaries start a certain mobility, fluctuations around the mean value of entropy are random for all practical purposes (differences between small numbers amplify the randomness of their queues): this is the meaning of the first valley for $2<T<T_{1}$.

\section{Results}

Let's now investigate the thermal behavior of "geometrical" quantities like Shannon entropy, Rohlin distance $d_{R}$, Hamming distance $d_{H}$, their standard deviations (SD) and spectral properties. 


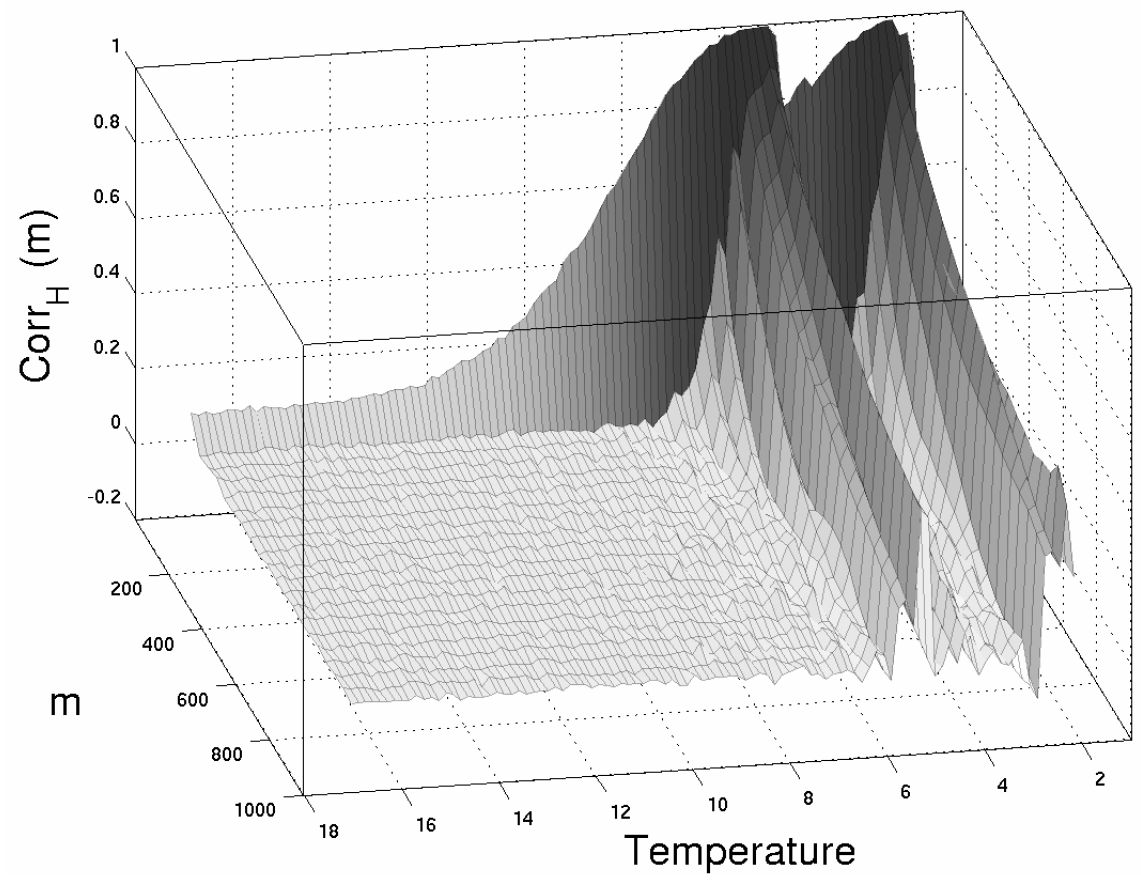

Figure 4: Entropy Correlation, $L=32$, mean values over four i.c.

Shannon entropy vs. temperature is shown in Fig.5. At low temperature, stable stripes of width $h=8$ lead correctly to $H=\ln 4$ for $L=32$ and $H=\ln 8$ for $L=64$. The transition around $T_{1}$ is quite clear. More precisely, when stripes begin to melt into two connected macro clusters, a drop is seen compatible with appearance of small spots (spins pointing in the opposite direction with respect to the background). In other terms, the Shannon entropy gives a quantitative evidence to the breakdown of the ground state connected domains, which discontinuously changes the cluster measure distribution by joining stripes into macro clusters. The example of Fig.3 clearly refers to this intermediate situation, when stripes still exist during long time intervals in an almost steady status with small oscillations at the borders, but may also suddenly melt or separate, modifying the cluster measures.

The regular increase of $H$ for $T>6$ indicates a progressive fragmentation of the macro clusters, or the growing relevance of islands, but nothing can be said about $T_{2}$. As to the nature of this fragmentation, the onset of some kind of fractality for greater $L$ cannot be excluded. However, for comparison, we recall that the observed fractal fragmentation around the transition temperature in the NN Ising ferromagnet gives a sudden change of concavity, with vertical inflexion point, exactly at $T_{c}$. In conclusion, where the transition for PFD is confirmed (at $T_{1}$ ), there is no fractality, and where fractality could be possible (around $T_{2}$ ) there is no transition: in both cases the difference with respect to the NN Ising model is clear.

Also the Entropy standard deviations (or ESD), calculated for each temperature along the orbits, point out a critical behavior around $T_{1}$, followed by a regular behavior for greater $T$, as shown in Fig.6. The peak at $T_{1}$ may be interpreted as due to time instability in the phase of melting stripes, corresponding indeed to intermittent behavior in the melting process of clusters (as illustrated by Fig.3). This phase is followed by the stabilization 


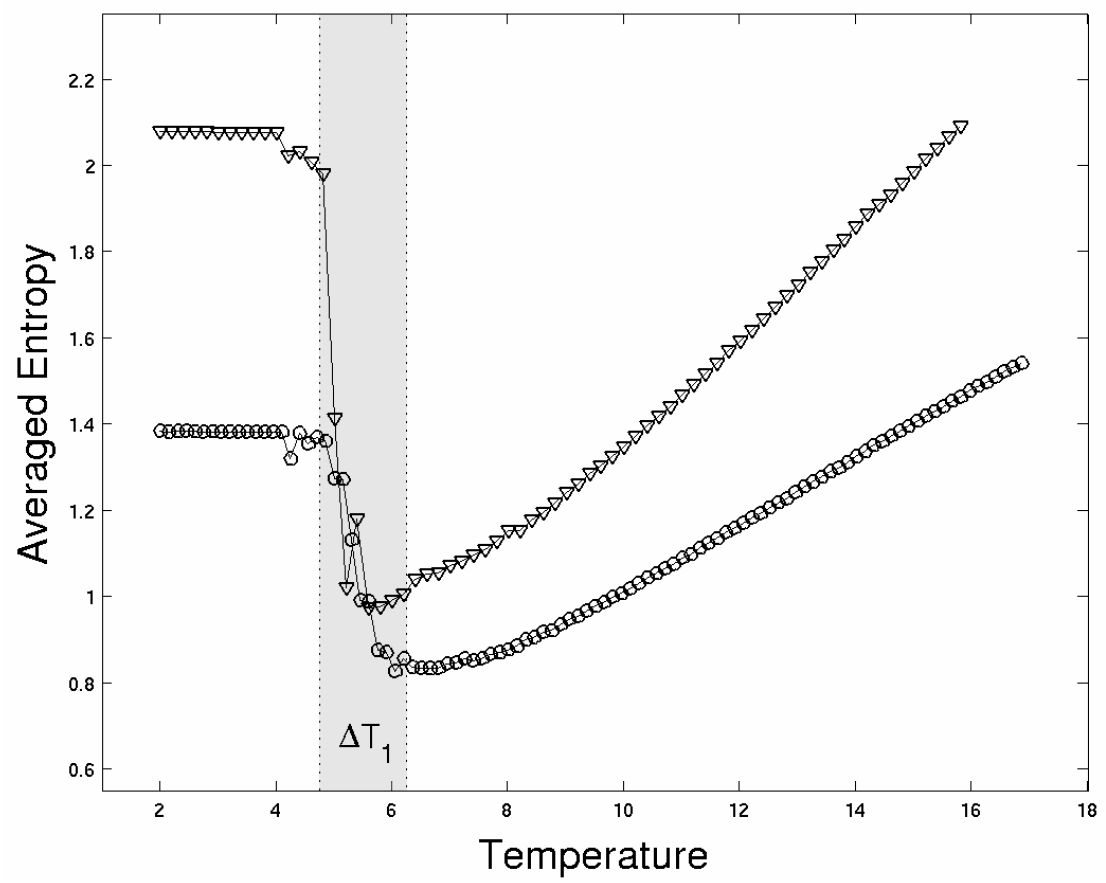

Figure 5: Time averaged entropy, for $L=32$ (circles) and $L=64$ (triangles), mean values over four i.c..

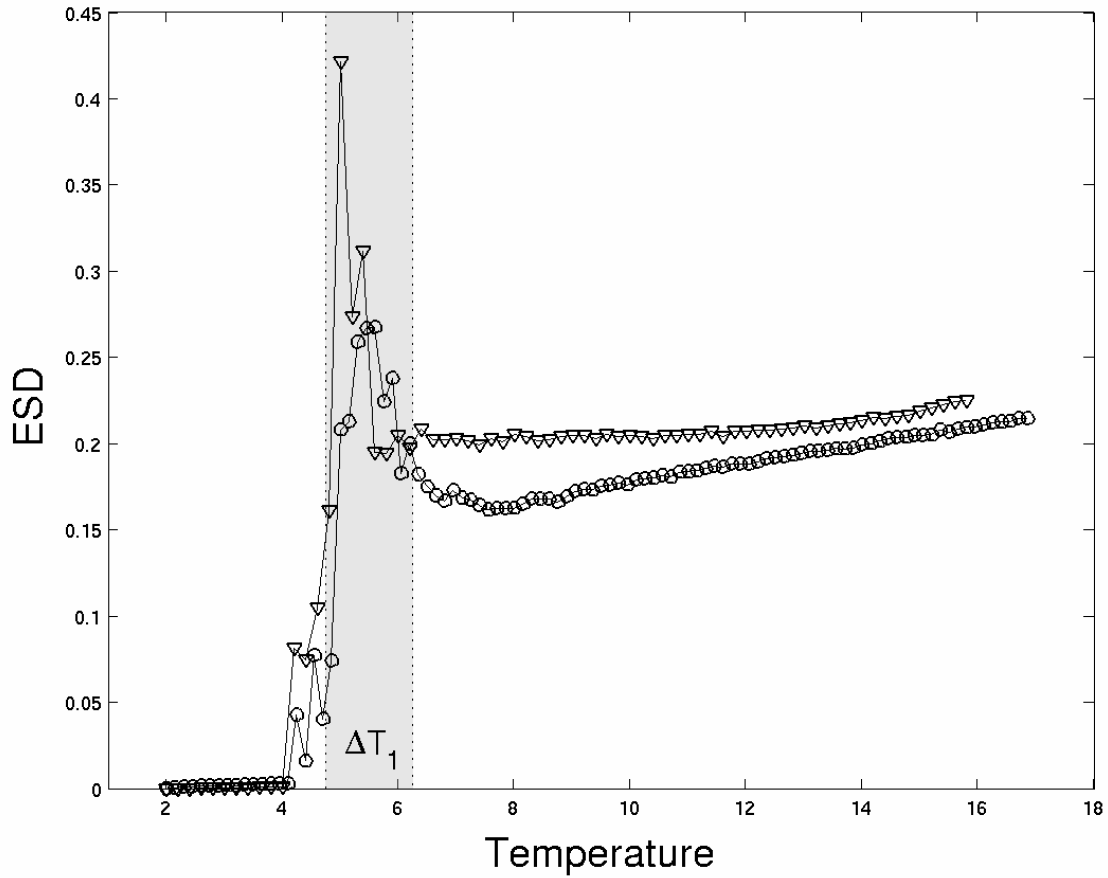

Figure 6: Entropy Standard Deviation in time (ESD) vs. temperature, same parameters and markers as in Fig.5. 
of the macro clusters (relative minimum of ESD). A new source of time instability is due to the cluster fragmentation, with appearance of islands, but once again this processes is smooth with respect to temperature, and no new transition can be recognized at $T_{2}$.

Fig.7 shows the Rohlin distance standard deviation (or RSD) versus temperature for three lattice sizes. The transition at $T_{1}$ is not clear, even if a singularity (discontinuity of

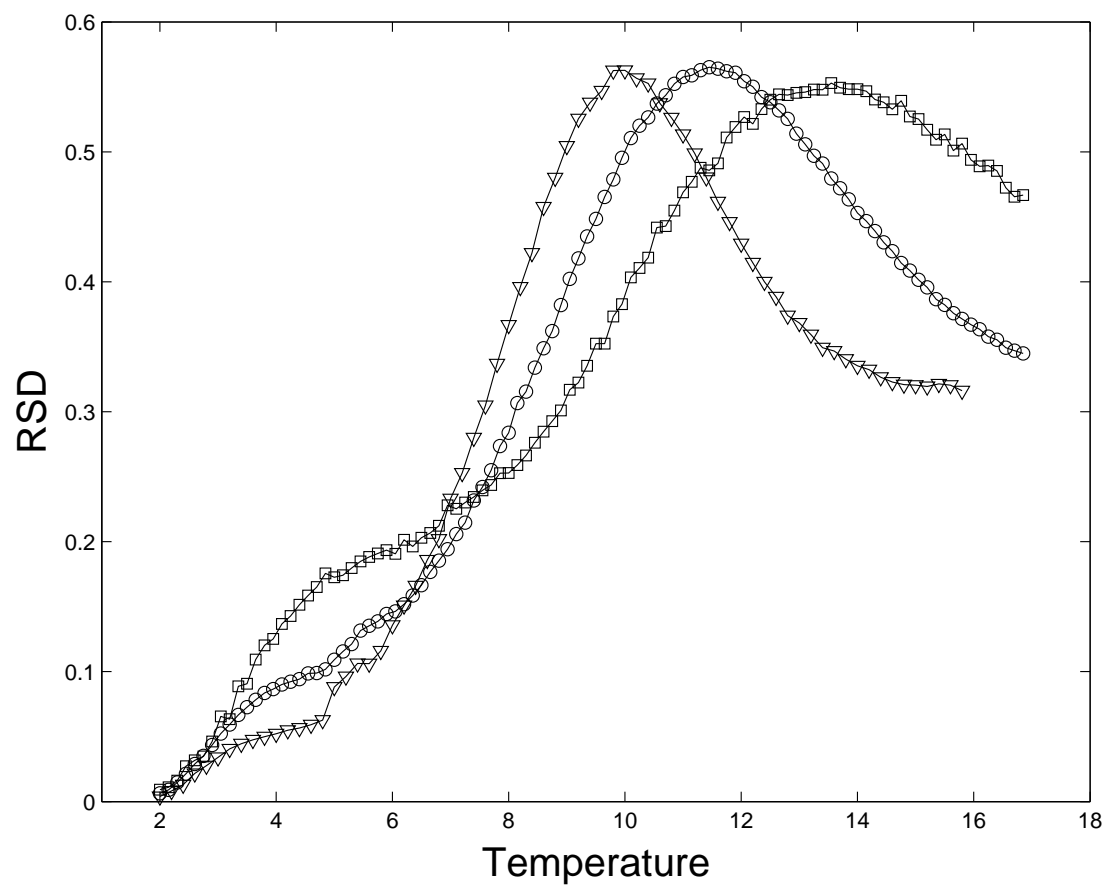

Figure 7: Rohlin distance Standard Deviation (RSD) in time vs. temperature, data for $L=16$ (squares), $L=32$ (circles) and $L=64$ (triangles), four i.c..

the first derivative) seems to develop at increasing $L$. A maximum occurs at a temperature not far from $T_{2}$, where the RSD shows an inflexion point. It is noteworthy that the value of the maximum is independent of $L$. When compared to the behavior of the $C_{V}$ peak, the behavior of this maximum vs. $L$ is surely different. Therefore, no correlation with a new transition can be recognized. We may see maximum of RSD in Fig.7 as the watershed between two opposite tendencies: 1) increase of time instability, due to the rising importance of islands with respect to macro clusters, and 2) the saturation of the phenomenon when macro clusters give up and the ensemble of disordered islands fill the lattice. In such a slow approach to chaoticity, the RSD decreases, as expected on the basis of previous experience on other models [4], where the maximum was a balancing point between fractal and chaotic configurations. In the present case, it would be hard to point out effective fractality because of small lattice sizes. As already noticed with entropy, a fractal phase during the fragmentation of macro clusters and the growth of islands remains only a reasonable conjecture, compatible with the observed behavior of RSD.

The Hamming distance is insensitive to the cluster shape, therefore it is not surprising that this kind of phenomena does not appear in its standard deviation (HSD), as shown in Fig.8. On the contrary, the occurrence of a singularity at $T_{1}$ may be sensed again for increasing $L$. Data in time series have been rescaled by $L$, leading to a good data collapse after $T_{1}$. This is consistent with the fact that, in the same range of temperatures, mean 


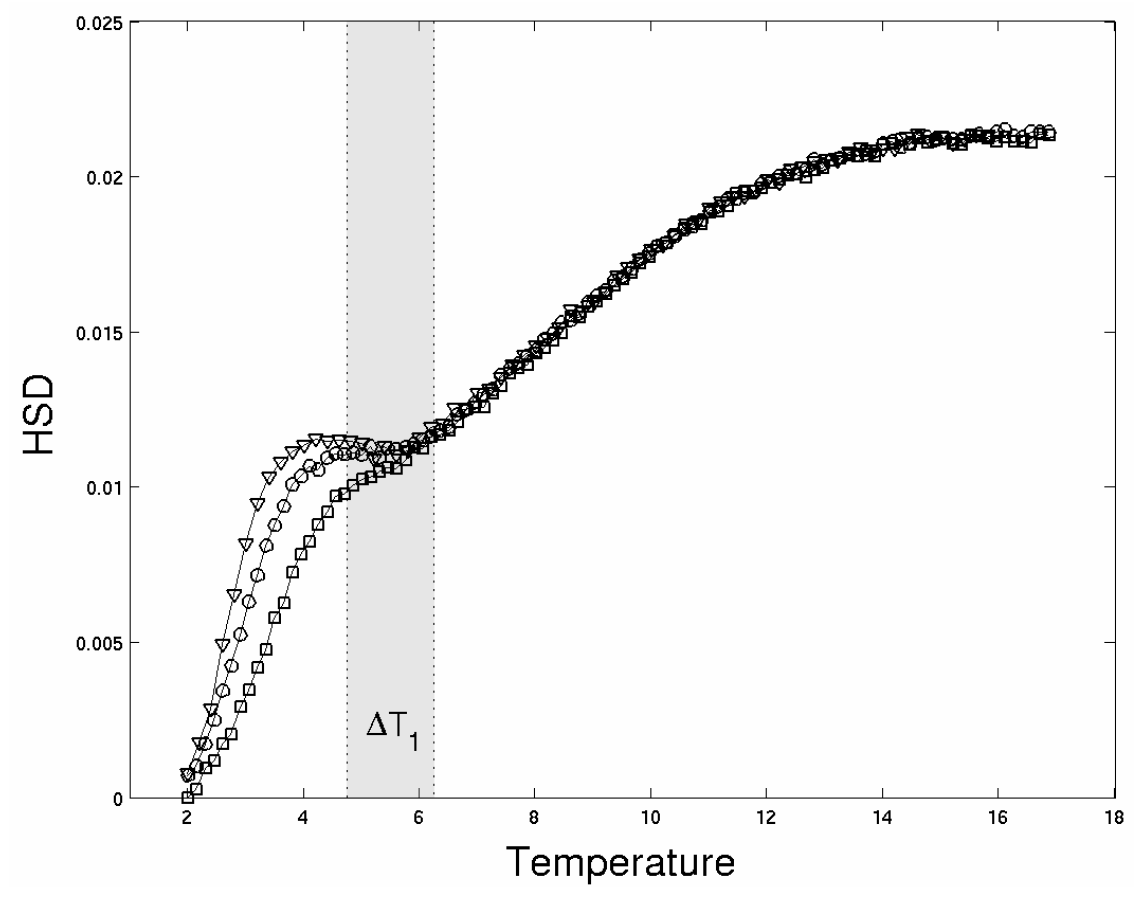

Figure 8: Hamming distance Standard Deviation (HSD)for PFD model, with $L=$ $16,32,64$. The time series have been rescaled by $L$. Same parameters and markers as in Fig.7.

values rescale with $N$. Moreover, such a data collapse seems to exclude that something may occur at $T_{2}$ for greater $L$.

A comparison with the corresponding behavior in the Ising ferromagnet (Fig.9) is instructive: there, the $L$-scaling behavior occurs indeed everywhere except around the transition temperature $T_{c}$, where also there is an incoming cusp that asymptotically in $L$ seems to get an infinite derivative (in this case we reach $L=100$ ). For both models, HSD behave qualitatively as the standard deviations of the total cluster perimeters (we omit to report figures). Since the perimeter SD is due to boundaries fluctuations, such a coincidence seems to indicate a sensitivity of the Hamming distance to the boundaries instability. A parallelism of this kind is not obvious, considering the different role of boundaries: because of long range interactions, indeed, the evolution rule in PFD model does not assign to borders the same importance as in Ising model.

Spectral features: by Fast Fourier Transform on time series, we obtained power spectra ( a typical example for Entropy is shown in Fig.10). As it is well known, there is no general dynamical theory on the presence of colored noise in signal sources, in front of an extremely rich phenomenology (see e.g. [14] [15]). In our context, experience on comparable time series for other models (Ising ferromagnet and SOC) confirms the widely discussed empirical link between fractality and colored noise, provided that the lattice size is sufficient to achieve a reasonable fractality [3] [4][5]. Therefore, in the present case, such a link remains conjectural, but this makes the observation of the noise even more interesting.

As a general feature, for all observables there is in fact the expected tendency to chaoticity as $T$ increases, but at $T=16$ a genuine white noise regime is not yet achieved. This 


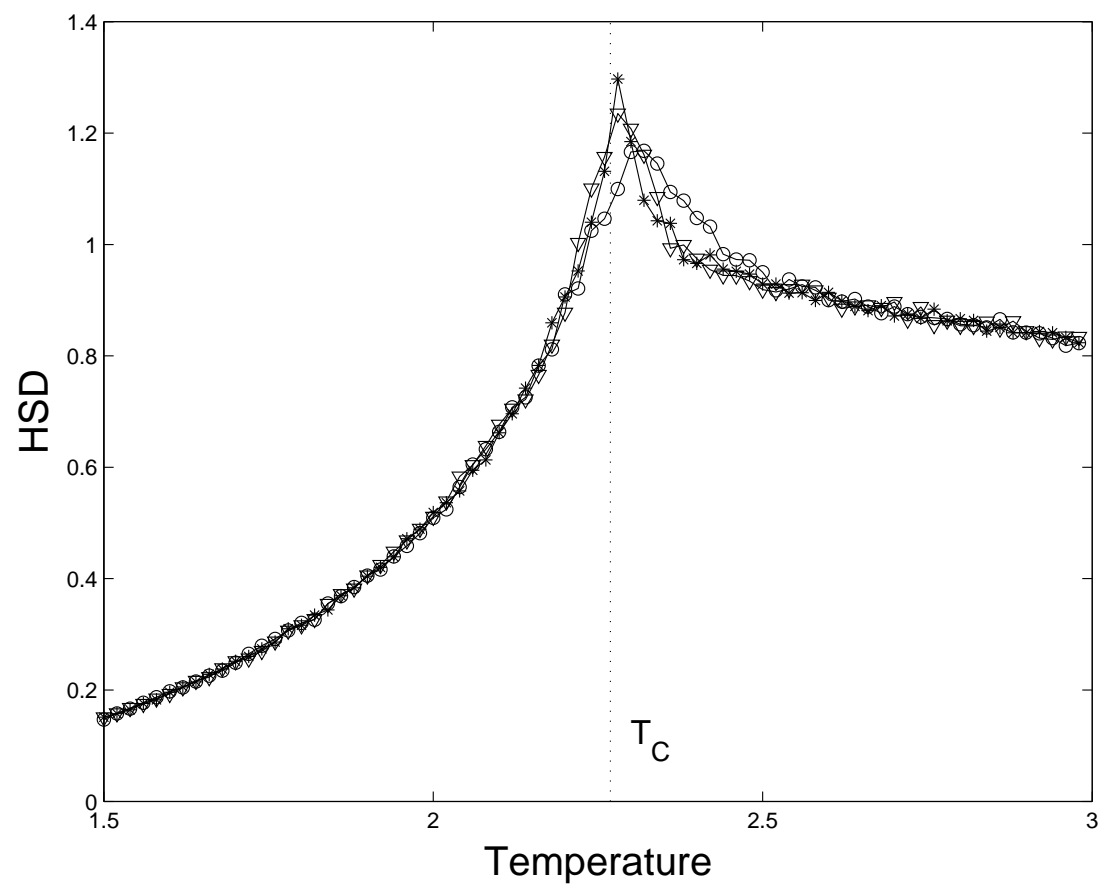

Figure 9: Hamming distance Standard Deviation for Ising ferromagnetic model, with $L=$ 32, 64, 100 (circles, triangles and stars respectively). Time series have been rescaled by $L$.

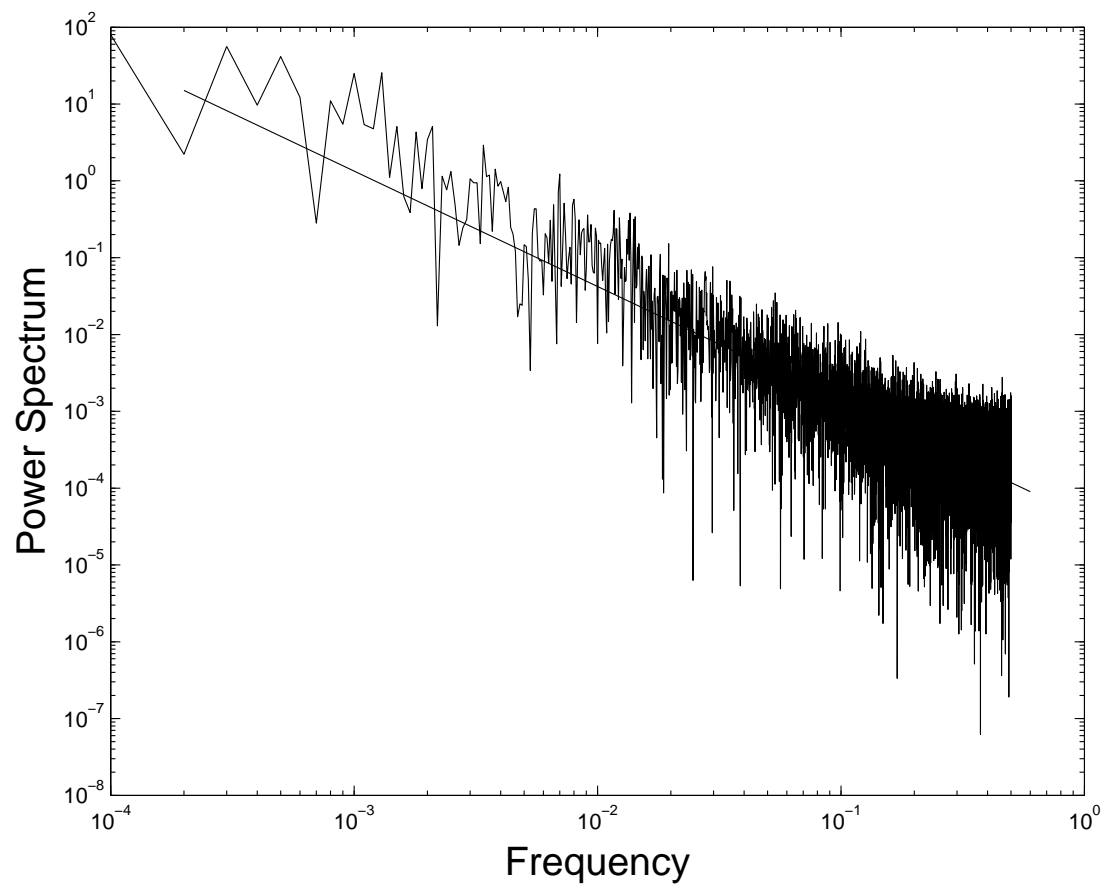

Figure 10: Power Spectrum and linear fit, an example on entropy time series, $T=5.4$. 
agrees with the disordered but not completely chaotic aspect of fragmented clusters at the same temperature. Thus, in the whole range of interest, colored noise $\omega^{\alpha}, \alpha<0$, is the rule. This exponent can be obtained as the value of the angular coefficient from the linear fit in the loglog plot of the power spectrum. For instance, Fig.10 shows the loglog plot od the power spectrum of the Shannon entropy for $T=5.4$. The linear fit gives $\alpha=-1.54$. A simple way to get information on the dependence of the noise is to plot the exponent $\alpha$ vs. T. For entropy spectra, the result is exhibited in Fig.11. Here we preferred not to

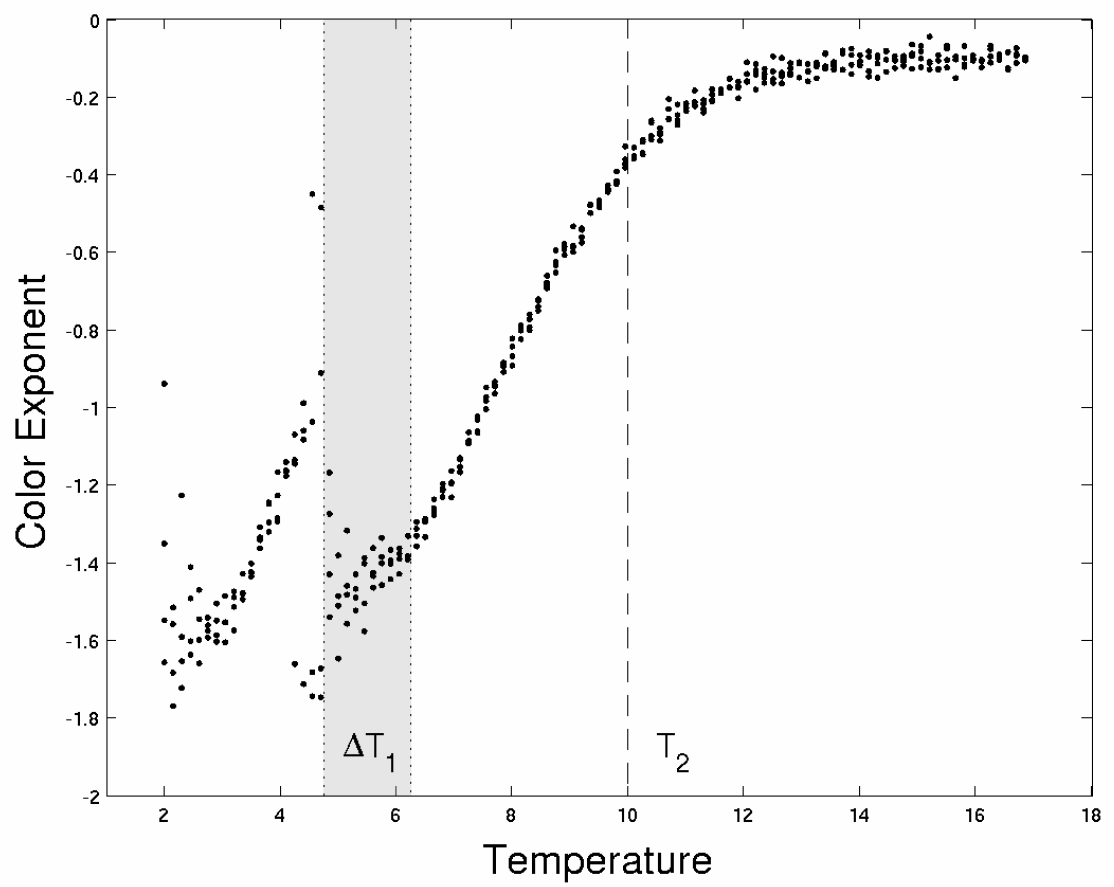

Figure 11: Color Exponents $\alpha$ from Entropy Spectra, four distinct i.c. at each temperature.

average over different i.c., in order to stress the dispersion of values in the critical interval $\Delta T_{1}$. The maximum spread coincides with the beginning of the interval $\Delta T_{1}$. Then, after the well (coinciding with the onset of macro clusters) there is the expected slow growth, up to values close to 0 from below. It is instructive to consider the analogous figure of the spectral exponent for the NN Ising model (Fig.12). This figure does not exhibit any burst of instability around the critical temperature $T_{c}=2.27 \mathrm{~J}$, where a phase transition characterized by a fractal structure of clusters occurs [4]. Moreover, the minimum is higher $(-1.2$ vs. -1.6). The qualitative difference between cluster geometry is therefore well reflected by Figs.11-12. More precisely, in the present case, the slow dynamics of small deformation of stripes $(\alpha \simeq-1.5)$ is followed by a substantial acceleration when domains start to merge. At $T_{1}$, temperature marking the appearance of very unstable stripes, dynamics slows down again. This pattern is confirmed also by Fig.4, by identifying slow/fast dynamics with long/short memory of correlations. Above $T_{1}$, the growth toward white noise is continuous: it does not give any particular relevance to $T_{2}$, possibly apart from a pseudo-fractality of very different nature with respect to the fractality found in NN Ising model. The exponents to compare are indeed $\alpha \simeq-1.2$ (Ising) vs. $\alpha \simeq-0.4$ (PFD). We stress again that this comparison is not between exponents at comparable temperatures, i.e. at the transition, but between the "more fractal patterns" in the two models (effective in a case, virtual in the other). 


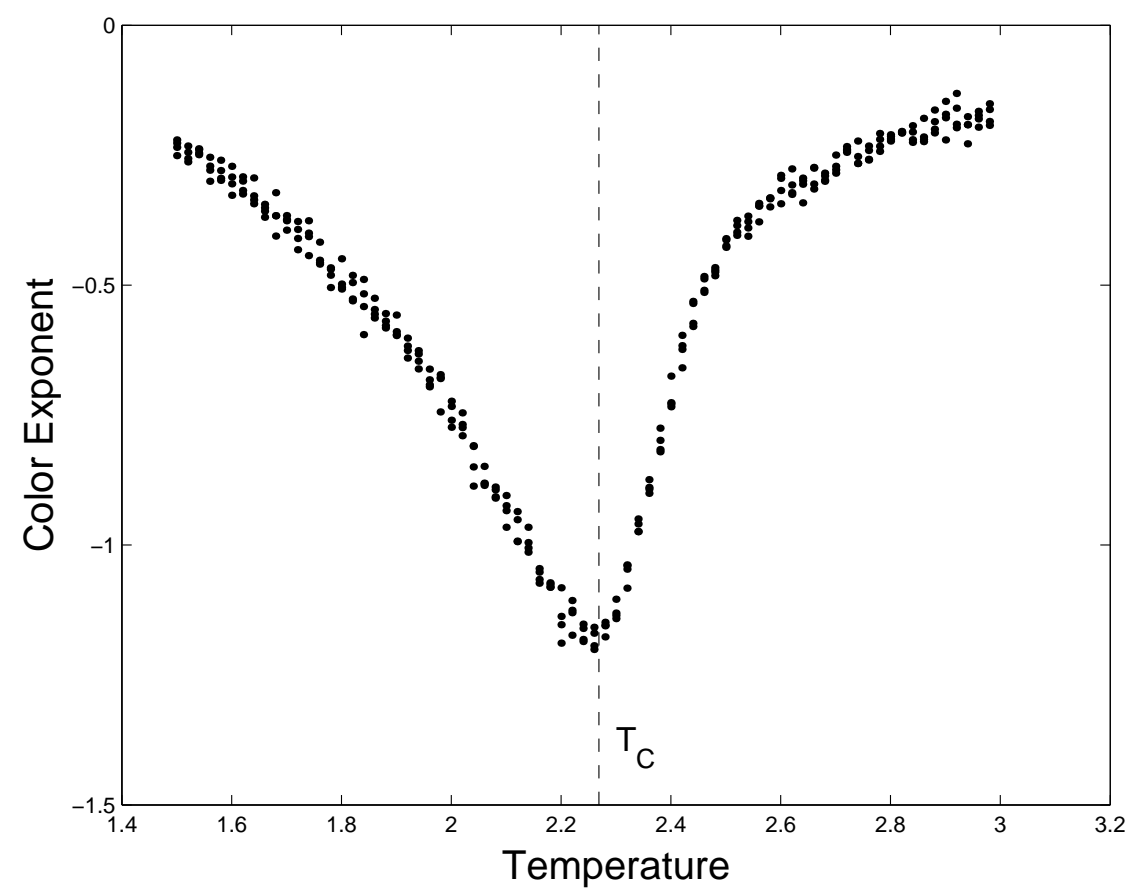

Figure 12: Color Exponents from Entropy Spectra, Ising system, four distinct i.c. at each temperature.

Some additional considerations on the thermal aspects of previous results could be useful. As it is well known, the specific heat may be calculated as

$$
C_{V}=\frac{<H^{2}>-<H>^{2}}{N k_{B} T^{2}}
$$

$k_{B}$ being the Boltzmann constant (here $k_{B}=1$ ). Labelling the exchange and the dipolar contributes in the Hamiltonian (1) by indexes $e$ and $d$ respectively, we write:

$$
C_{V}=\frac{<H_{e}^{2}>-<H_{e}>^{2}}{N k_{B} T^{2}}+\frac{<H_{d}^{2}>-<H_{d}>^{2}}{N k_{B} T^{2}}+2 \frac{<H_{e} H_{d}>-<H_{e}><H_{d}>}{N k_{B} T^{2}} .
$$

The first two terms in the r.h.s., we denote $C_{e}$ and $C_{d}$, have the form of a specific heat for the exchange and dipolar Hamiltonian respectively (of course, they are not!). The last term, say $C_{e d}$, is a sort of correlation. Since $C_{V}=C_{e}+C_{d}+C_{e d}$, one may look for the origin of peaks at $T_{1}$ and $T_{2}$ by observing separately $C_{e}+C_{d}$ and $C_{e d}$. Actually, both these quantities give neat evidence of a peak at $T_{1}$, and no evidence at all of a peak at $T_{2}$, as shown in Fig.13, where a close correlation between $C_{e}+C_{d}$ and $C_{e d}$ appears in the whole range. Moreover, the ratio $\left(C_{e}+C_{d}\right) / C_{e d}$, given in Fig.14, indicates quite clearly that there exist two distinct regimes of proportionality, $T_{1}$ being once again the turning point of them.

All conspire in saying that $T_{2}$ has no thermodynamic relevance. This conclusion completely agrees with the geometrical characterization suggested by entropy and distances: the maximum of $C_{V}$ at $T_{2}$ seems to indicate a balancing point between growing and decreasing contributes related to the smooth fragmentation process of clusters. 


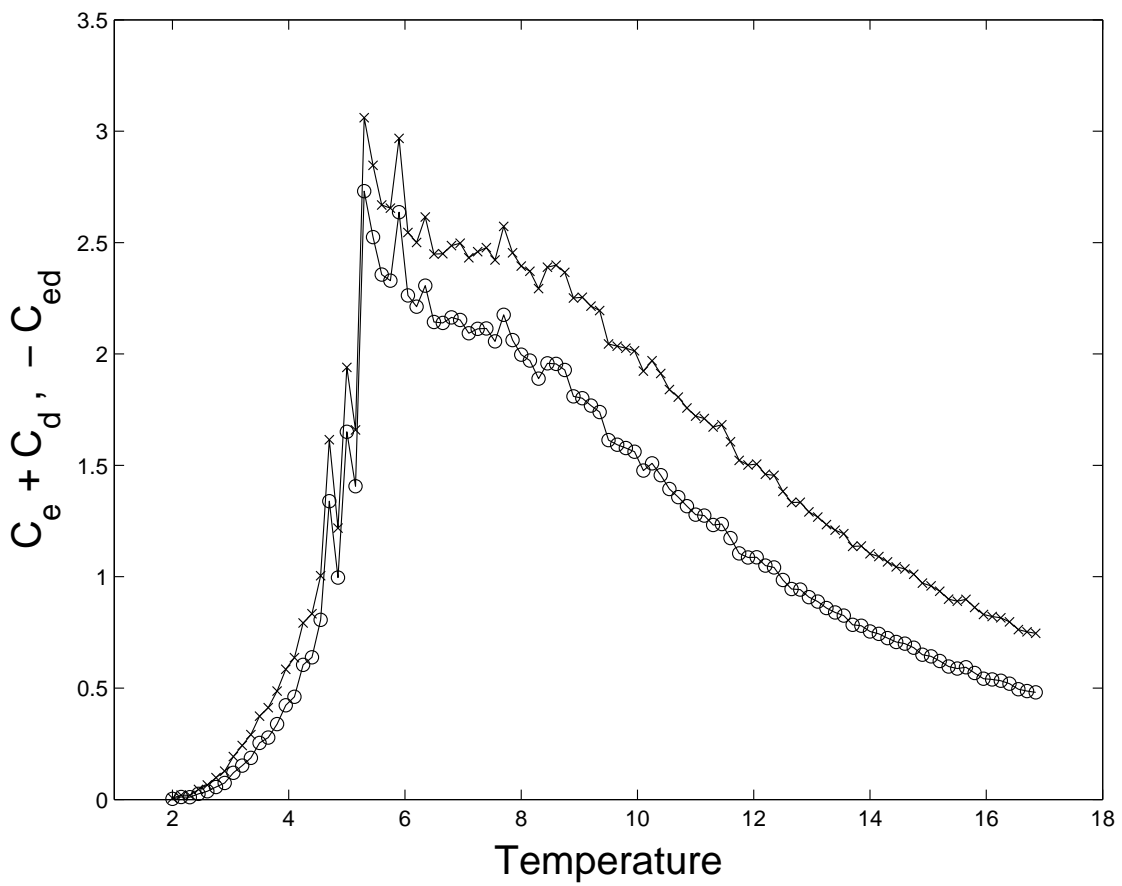

Figure 13: Contributes to the specific heat: $C_{e}+C_{d}$ (crosses), $-C_{e d}$ (circles), mean values over four i.c..

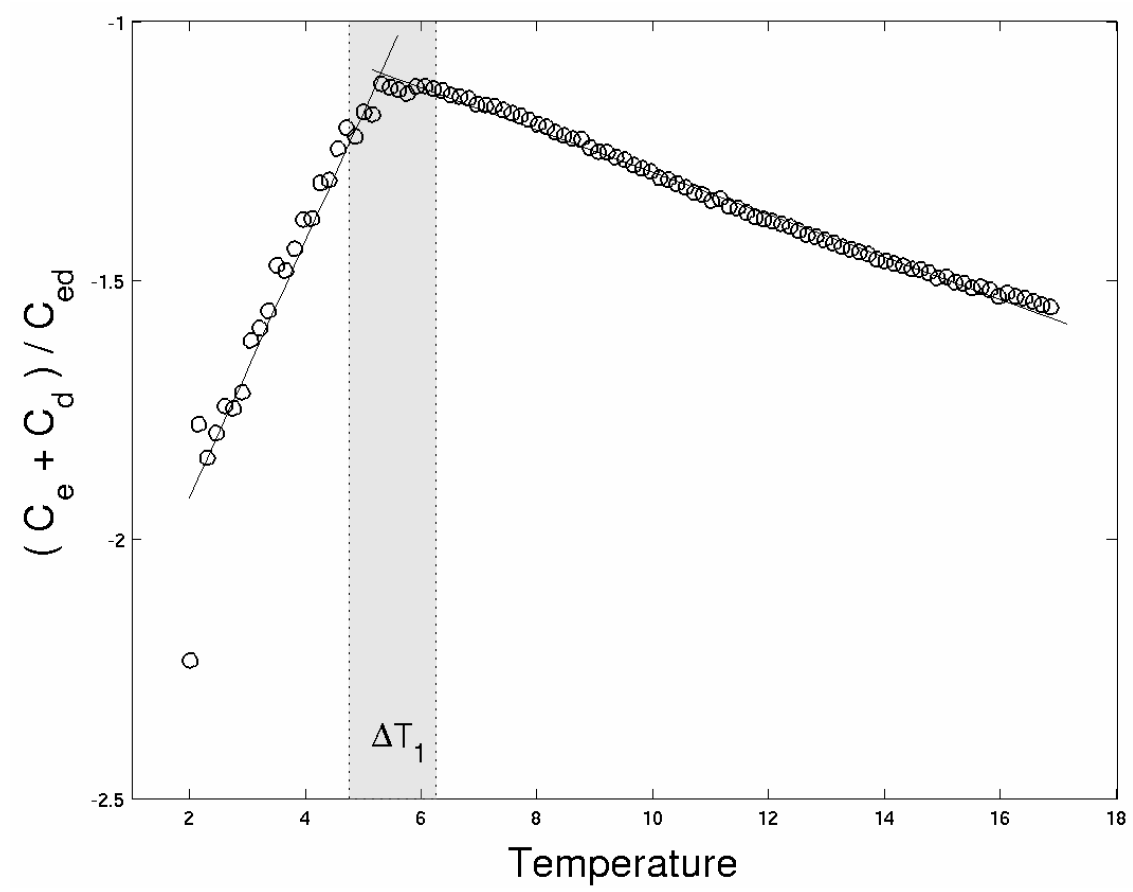

Figure 14: Ratio $\left(C_{e}+C_{d}\right) / C_{e d}$, same parameters as in Fig.13. 


\section{Previous results revisited}

We recall that previous authors, in order to evaluate the orientational symmetry of the striped states, introduced two domain order parameters, $O$ and $\eta$.

In the dual lattice, let $n_{h}$ and $n_{v}$ be the number of horizontal and vertical sides along the cluster boundaries ( $n=n_{h}+n_{v}$ is therefore the total border length, or total perimeter). The first parameter $O$, introduced in [1], is the time averaged difference

$$
O=\left\langle\frac{n_{h}-n_{v}}{n}>\right.
$$

It estimates the deviation from an isotropic distribution of sides in the clusters. In the purely striped domain, there are only horizontal or vertical sides, so that $O= \pm 1$ (depending on the initial orientation), while the parameter must be 0 in an isotropic configuration. Isotropy is expected to hold not only in the disordered phase at high temperature, but just after the stripes breakdown. What one actually sees in Fig.15, starting e.g. with $O=1$ for $T<2$, is that the parameter weakly decreases up to temperatures where a preferential orientation clearly persists. But the subsequent transition to 0 , just around $T_{1}$, is not smooth at all, presenting a remarkable oscillation of sign, as if the remainder of the stripes suddenly changed orientation for long time intervals. For all $L$, the sign oscillation interval coincides with the peak interval $\Delta T_{1}$ of the specific heat. For both $C_{V}$ and $O$, this interval is expected to narrow in the thermodynamic limit. Except for these fluctuations around the value zero

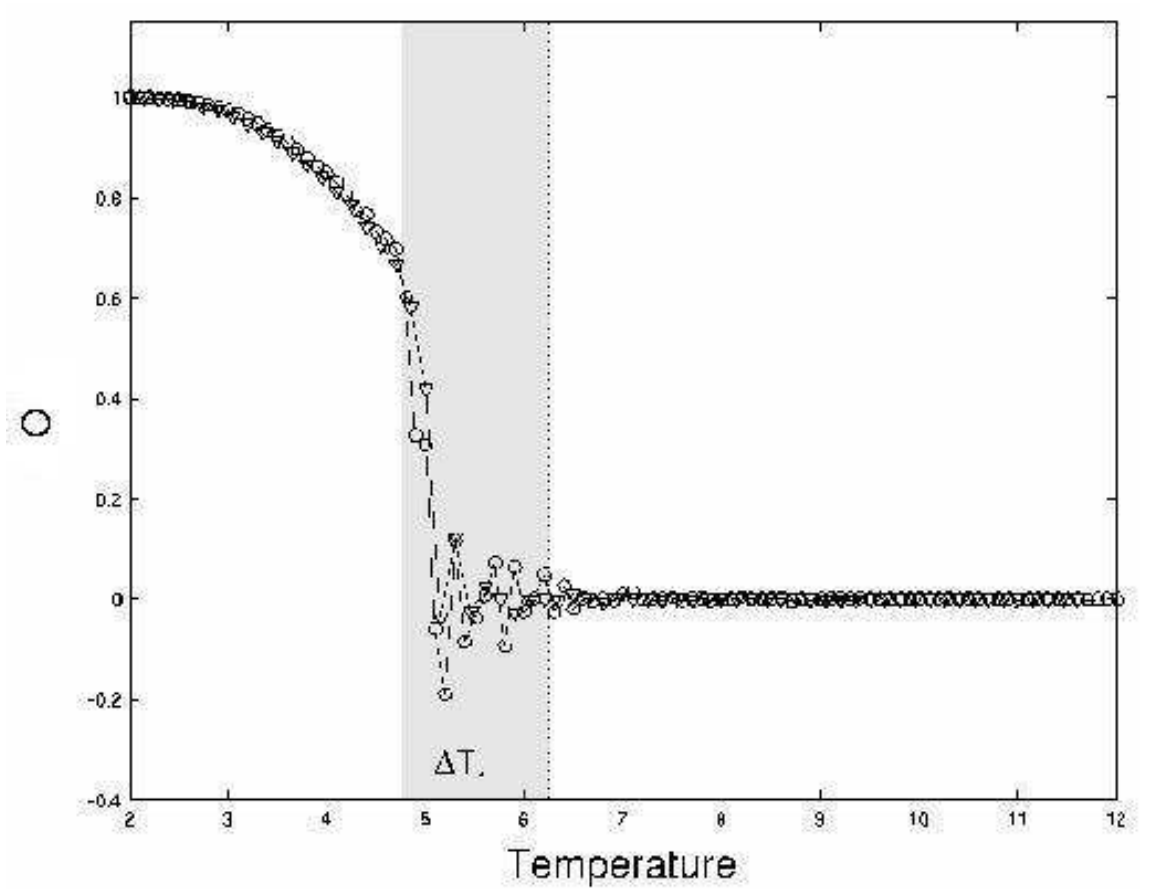

Figure 15: Parameter $O$, or first domain order parameter, for $L=32$ (circles) and $L=64$ (triangles), mean values over four i.c..

in the critical region, Fig. 15 recovers Fig.4 of [1], apparently built up with the absolute values of the parameter. Even if such inversions were a finite size effect, as suggested by 
the amplitude of oscillations decreasing with $L$, this attitude is a remarkable signature of the way the stripes collapse in the transition region. This oscillatory phenomenon could be analogous to the magnetization inversion observed in the NN Ising model, for finite size lattices, when fractal patterns begin to take place approaching the transition temperature. Note that in PFD this orientation incertitude takes place mostly after $T_{1}$, in a range of temperatures where stripes are still melting into two big clusters with small fragments, as shown by the entropy.

We recovered also the parameter $\eta$. Assuming $s_{i, j} \equiv s_{\mathbf{k}}$ the spin variable, $\eta$ is defined by formula $(2)$ in $[2]$, i.e.:

$$
\eta=\frac{1}{N}\left\langle\left|\sum_{j}\right| \sum_{i} s_{i, j}\left|-\sum_{i}\right| \sum_{j} s_{i, j}||\right\rangle .
$$

Also this parameter is proven to be effective in detecting the first temperature (see. Fig.3 in [2]), but it cannot capture the oscillatory phenomenon revealed by $O$.

\section{Conclusions}

The observation of cluster dynamics and the related statistical properties give clear evidence to the following points:

1. the peak for $C_{V}$ at $T_{1}$ is correlated to a melting process of stripes without any occurrence of fractality, a behavior quite different with respect to the NN Ising model;

2. some analogy between the two models seems to exist in the attitude to sudden inversion of stripe orientation or magnetization respectively, as finite size effects. Such an analogy does not imply any similitude in the cluster geometry: in one case, the orientation anisotropy keeps a memory of the stripped structure, while in the other case the inversion of magnetization has to do with the onset of fractality at $T_{c}$;

3. colored noise is present in both models at their transitions (Figs.11-12), in a quite different fashion: in the PFD case, with a wide range of values as a consequence of intermittent melting phenomena with small perturbations at boundaries (see e.g. Fig.3), making spectra at $T_{1}$ still dependent on i.c. (this intermittency may be read also in the entropy standard deviation); in Ising, as a counterpart of fractal dynamics, almost independent of i.c. at $T_{c}$;

4. standard deviations for Rohlin and Hamming distances are different too in PFD and Ising systems. However, in both models, the Hamming SD have the same scaling behavior of the total perimeter;

5. as to the second peak shown in the $C_{V}$ diagram, only the SD of the Rohlin distance presents some peculiar behavior in the neighbors of $T_{2}$. It seems that dynamical regime at such temperatures corresponds to the fragmentation of macro clusters. Only here there is a possibility for fractal configurations, even if not detectable at the values of $L$ accessible to computations. 
In conclusion, we have a good evidence from geometry that the Ising ferromagnetic transition at $T_{c}$ and the PFD transition at $T_{1}$ are of different nature. As to $T_{2}$, on the basis of our geometric and dynamical indicators it cannot be recognized as a transition temperature at all. Consistently with expectations, we cannot therefore extend to our case the conjecture proposed in [12] on the irrelevance of the long range interactions for the Ising antiferromagnet class of universality.

\section{Acknowledgments}

We thank A. Tassi (Parma) for very useful discussions on the subject. 


\section{Appendix}

Let $\mathbf{M}$ be a $L \times L$ square lattice, where $\operatorname{knots}(i, j)$ assume values in an alphabet $\mathbf{K}$. A state or configuration on $\mathbf{M}$ is a whole set $\mathbf{a}=\left\{a_{i, j}\right\}, a_{i, j} \in \mathbf{K}$. It is an element of $\mathcal{C}=\mathcal{C}(\mathbf{M})$, the set of all $|\mathbf{K}|^{L \times L}$ possible states of the lattice. For instance, $\mathbf{K}=\{0,1\}$, fits the description of Ising-like systems. The dual lattice (we shall equally denote $\mathbf{M}$ ) is a $L \times L$ set of square cells corresponding to the knots.

When the alphabet $\mathbf{K}$ itself is a metric space (e.g. a numerical set with the usual $|x-y|$ distance), one can consider in $\mathcal{C}(\mathbf{M})$ the Hamming distance $d_{H}$ which, for configurations a and $\mathbf{b}$, is defined by the functional

$$
d_{H}(\mathbf{a}, \mathbf{b})=\sum_{i, j}\left|b_{i, j}-a_{i, j}\right|
$$

We stress that the Hamming distance is sensitive only to actual values of corresponding knots, not to their distribution or neighborhood.

A path, is a sequence of "near" knots, equivalent to a sequence of cells having common sides in the dual description. A connected cluster is a set of knots with the same value in $\mathbf{K}$ which are connected by a path. In the dual lattice, clusters are connected but not necessarily simply connected sets, made up of square cells. Since every cell belongs to a single cluster, clusters $A_{k}$ are disjoint subsets of $\mathbf{M}$ and $\bigcup_{k} A_{k}=\mathbf{M}$. In other terms, the clusters collection is a "finite partition" of $\mathbf{M}$. The subsets $\left\{A_{k}\right\}$ of a partition are often referred to as its "atoms". Let $\mathcal{Z}(\mathbf{M})$ denote the set of all finite partitions of $\mathbf{M}$. The correspondence $\Phi: \mathcal{C} \rightarrow \mathcal{Z}$ between a configuration a $\in \mathcal{C}$ and the clusters partition $\alpha \equiv\left(A_{1}, \ldots, A_{N}\right) \in \mathcal{Z}$, i.e. $\alpha=\Phi(\mathbf{a})$, is "many to one", since configurations generated by permutations in $\mathbf{K}$ are mapped into the same partition. If the cardinality of the alphabet is $|\mathbf{K}| \geq 4$, because of Euler's Four Colour Theorem for every partition $\alpha \in \mathcal{Z}$ there exist $\mathbf{a} \in \mathcal{C}$ with $\alpha=\Phi(\mathbf{a})$. This is not true for the case $\mathbf{K}=\{0,1\}$ considered in the present work.

A probability measure $\mu$ may be introduced in the algebra $\mathcal{M}$ of subsets of $\mathbf{M}$ : for every $A \in \mathcal{M}, \mu(A)$ is the normalized number of knots in $A$. Standard operations on partitions may be recovered in classical textbooks such as [8][9][10], or, for our demands, in [3][5]. Here we only recall the definition of Shannon entropy and Rohlin distance: Let $\alpha=\left(A_{1}, \ldots, A_{N}\right)$ be a partition: its Shannon entropy $H(\alpha)$ is

$$
H(\alpha)=-\sum_{i=1}^{N} \mu\left(A_{i}\right) \ln \mu\left(A_{i}\right) .
$$

Note that the Shannon entropy depends only on the cluster measures, not on their shapes. Shapes are taken into account by conditional entropy: if $\mathrm{f} \beta=\left(B_{1}, \ldots, B_{M}\right)$ is another partition, the conditional entropy of $\alpha$ with respect to $\beta$ is

$$
H(\alpha \mid \beta)=-\sum_{i=1}^{N} \sum_{k=1}^{M} \mu\left(A_{i} \cap B_{k}\right) \ln \frac{\mu\left(A_{i} \cap B_{k}\right)}{\mu\left(B_{k}\right)},
$$

and the Rohlin distance $d_{R}$ is

$$
d_{R}(\alpha, \beta)=H(\alpha \mid \beta)+H(\beta \mid \alpha) .
$$


This way, $\mathcal{Z}(\mathbf{M})$ is a metric space. The Rohlin distance between two finite partitions expresses how different they are. We also recall that there exists a method, called "reduction process ", to amplify as far as possible the non-similarity between partitions. This method is reminiscent of cancellation of common factors between integers, justifying the concept of "rational partitions" introduced in this context ([3][4]). However, for the model studied in the present work, the reduction process proves to be unimportant, and we shall disregard on it.

Hamming and Rohlin distances are not directly comparable. We stress that the Hamming distance is between configurations and it is sensitive only to actual values of corresponding knots, not to their distribution or neighborhood, whereas the Rohlin distance is between partitions, and therefore is sensitive to the cluster shapes. In principle, $d_{R}$ and $d_{H}$ may give very different information. With the binary $\{0,1\}$ alphabet, for instance, complementary configurations have maximal Hamming distance $\left(d_{H}=N\right)$, while the corresponding partitions coincide $\left(d_{R}=0\right)$.

If a configuration $a \in \mathcal{C}$ has discrete evolution $a, T a, T^{2} a, \ldots$, one can speak of "configurations orbit". The corresponding dynamics $\hat{T}$ on $\mathcal{Z}$ is defined by

$$
\hat{T} \alpha=\hat{T} \Phi(a)=\Phi(T a)
$$

so that to a configurations orbit there corresponds a partitions orbit. Clearly, the probability measure $\mu$ in $\mathcal{M}$ is not preserved by the evolution, in the sense that clusters or atoms are redefined at every step by the pointwise evolution, and do not evolve in themselves. However, we are not interested here in such indicators as Kolmogorov-Sinai entropy or Lyapunov exponents, requiring a preserved measure. Observables $F$ are defined at each time in $\mathcal{C}(\mathbf{M})$ or $\mathcal{Z}(\mathbf{M})$, and they give rise to "time series" $\left\{x_{k}\right\}=\left\{F\left(T^{k} a\right)\right\}$ or $\left\{x_{k}\right\}=\left\{F\left(\hat{T}^{k} \alpha\right)\right\}$. Such time series are the main objects of our investigations. Typically, we shall consider

- $x_{k}=H\left(\hat{T}^{k}(\alpha)\right)$, i.e. the entropy time series;

- $x_{k}=d_{R}\left(\hat{T}^{k}(\alpha), \hat{T}^{k-1}(\alpha)\right)$, i.e. the Rohlin distance time series;

- $x_{k}=d_{H}\left(T^{k}(a), T^{k-1}(a)\right)$, i.e. the Hamming distance time series.

This formalism applies in principle to every kind of lattice and discrete dynamics, and could be easily extended to graphs. However, a computational obstacle consists in the necessity of handling the cluster borders, a difficult task for large lattice sizes (and even more in dimension $d>2$ ). 


\section{References}

[1] I. Booth, A. B. MacIsaac, J. P. Whitehead and K. De Bell, Phys. Rev. Lett. 75 (1995) 950-953.

[2] M. Ifti, Q. Li, C.M. Soukolis and M.J.Velgakis, Modern Phys. Lett. B15, (2001) 895903.

[3] M.Casartelli : Complex Syst. 4 (1990) 491-507; A. Albrigi and M. Casartelli: Complex Syst. 7 (1993) 171-197

[4] D.Bettati, M.Casartelli, P.Celli and L.Malpeli: J. Phys A: Math. Gen. 31 (1998) 93599376.

[5] M.Casartelli and M.Zerbini: J. Phys. A: Math. Gen. 33 (2000) 863-872.

[6] R. Peierls Proc. Cambridge Phil. Soc. 32 (1936) 477; R. B. Griffith Phys. Rev. A136 (1964) 537.

[7] R.Kretschmer and K. Binder, Z.Phys.B. Condens. Matter 34 (1979) 375.

[8] P. Billingsley: Ergodic Theory and Information, Wiley (1965).

[9] V.I.Arnold and A. Avez: Problèmes Ergodiques de la Mécanique Classique, (GauthierVillars, Paris 1967).

[10] I.P. Cornfeld, S. V. Fomin and Ya. G. Sinai: Ergodic Theory (Springer-Verlag, 1982).

[11] N. F. G. Martin and J, W. England: Mathematical Theory of Entropy, AddisonWesley, Reading MA (1981).

[12] A. B. MacIsaac, J. P. Whitehead, De Bell and K. Sowmya Narayanan: Phys. Rev. B46 (1992) 6387.

[13] N. Metropolis, A. W. Rosenbluth, M.N. Rosenbluth, A.H. Teller and E. Teller: J. Chem. Phys. 21 (1953) 1087; T. Toffoli and N. Margolus: Cellular Automata Machines (MIT U.P., Cambridge 1987).

[14] D. B. Percival and A. T. Walden: Spectral Analysis for Physical Applications, Cambridge U.P. (1993).

[15] M. Schroeder: Fractals, Chaos, Power Laws (W. H. Freeman and Company, New York 1991) 